\title{
Combined solid-state NMR, FT-IR and computational studies on layered and porous
} materials

Geo Paul, a,b Chiara Bisio,, ${ }^{\text {a,b }}$ Ilaria Braschi, ${ }^{\mathrm{b}, \mathrm{c}}$ Maurizio Cossi, ${ }^{\mathrm{a}, \mathrm{b}}$ Giorgio Gatti, ${ }^{\mathrm{a}, \mathrm{b}}$ Enrica Gianotti, ${ }^{\mathrm{a}, \mathrm{b}}$ Leonardo Marchese ${ }^{* a, b}$

\section{Electronic Supplementary Information (ESI)}

1. Acronyms, symbols and abbreviations

$\begin{array}{ll}\text { 1D } & \text { One-dimensional } \\ \text { 2D } & \text { Two-dimensional } \\ \text { 3D } & \text { Three-dimensional } \\ \text { TMP } & \text { 2,4,6-trimethylpyridine } \\ \delta & \text { chemical shift } \\ \delta_{\text {iso }} & \text { isotropic chemical shift } \\ \text { ADF } & \text { Amsterdam density Function } \\ \text { ADOR } & \text { assembly-disassembly-organisation-reassembly } \\ \text { AFM } & \text { Atomic force microscopy } \\ \text { ALPO } & \text { Aluminiumphosphate molecular sieve } \\ \text { APTS } & \text { 3-aminopropyltriethoxysilane } \\ \text { ATR-IR } & \text { Attenuated total reflection - infrared } \\ \text { BAS } & \text { Brønsted acid site } \\ \text { BEA } & \text { beta type zeolite } \\ \text { CHA } & \text { Chabazite type zeolites } \\ \text { CMP } & \text { conjugated microporous polymers } \\ \text { COF } & \text { covalent organic frameworks } \\ \text { CP } & \text { cross-polarization } \\ \text { CSA } & \text { chemical shift anisotropy } \\ \text { CTA } & \text { cetyltrimethylammonium } \\ \text { CTF } & \text { covalent triazine frameworks }\end{array}$




$\begin{array}{ll}\text { DAS } & \text { dynamic angle spinning } \\ \text { DFT } & \text { density functional theory } \\ \text { DQ } & \text { double quantum } \\ \text { DMF } & \text { N,N-dimethylformamide } \\ \text { DMFIT } & \text { a program for fitting NMR spectra } \\ \text { DRIFT } & \text { diffuse reflectance infrared spectroscopy } \\ \text { DOPA } & \text { dihydroxyphenylalanine } \\ \text { DOR } & \text { double rotation } \\ \text { DPE } & \text { deprotonation energy } \\ \text { EAPTS } & \text { 3-(2-aminoethyl)aminopropyltrimethoxysilane } \\ \text { EFAL } & \text { extra-framework aluminium } \\ \text { EFG } & \text { Electric field gradient } \\ \text { ESR } & \text { electron spin resonance } \\ \text { ETP } & \text { ethane-to-propene } \\ \text { EXAFS } & \text { Extended X-ray Absorption Fine Structure } \\ \text { FAL } & \text { framework aluminum } \\ \text { FAU } & \text { Faujasite type zeolite } \\ \text { FER } & \text { Ferrierite type zeolite } \\ \text { FT-IR } & \text { Fourier-transform infrared } \\ \text { HCP } & \text { hyper-cross-linked polymers } \\ \text { HDTMA } & \text { hexadecyltrimethylammonium } \\ \text { HETCOR } & \text { heteronuclear correlation } \\ \text { HMF } & \text { 5-hydroxymethylfurfural } \\ \text { HRTEM } & \text { high resolution transmission electron microscopy } \\ \text { ICP-ES } & \text { inductively coupled plasma emission spectrometry } \\ \text { IEZ } & \text { Interlayer expanded zeolitic materials } \\ \text { INEPT } & \text { insensitive nuclei-enhanced by polarization transfer } \\ \text { INS } & \text { inelastic neutron scattering } \\ \text { IR } & \text { infrared } \\ \text { KD } & \text { kinetic diameter } \\ \text { LAS } & \text { layered double hydroxides } \\ \text { Mobil Composition of Matter No. 41 }\end{array}$


MD molecular dynamics

MeAPO metal-aluminophosphate molecular sieve

MeAPSO metal-aluminophosphosilicate molecular sieve

MM molecular mechanics

MOF metal-organic frameworks

MOR mordenite type zeolite

MOP microporous organic polymers

MQMAS multiple quantum magic angle spinning

MTO methanol-to-olefins

MSN mesoporous silica nanoparticles

NBO natural bond orbital

NION nanoporous ionic organic networks

NMR nuclear magnetic resonance

ODTMA octadecyltrimethylammonium

OTS octadecyltrichlorosilane

PAF porous aromatic frameworks

PAPTS 3-[2-(2-aminoethyl)aminoethyl] aminopropyltrimethoxysilane

PBE Perdew-Burke-Ernzerhof

PDA pore directing agent

PFG pulsed field gradient

PIM polymers of intrinsic microporosity

PMM porous molecular materials

PMO periodic mesoporous organosilicas

PXRD X-ray Powder Diffraction

RB Rose Bengal dye

REDOR rotational echo double resonance

RT room temperature

SAP saponite clay

SAPO silicoaluminophosphate molecular sieve

SBA Santa Barbara Amorphous

SDA structure directing agent

SIMPSON simulation program for solid-state NMR

SOC spin-orbit coupling

SOD Sodalite type zeolite

SQ single quantum 
S-RESPDOR Symmetry-based Resonance-Echo Saturation-Pulse DOuble-Resonance

SsNMR solid-state nuclear magnetic resonance

STMAS satellite transition magic angle spinning

TBAOH tetrabutylammonium hydroxide

TBPO tributylphosphine oxide

TEPO triethylphosphine oxide

TGA Thermogravimetric analysis

TMA Tetramethylammonium

TMP trimethylphosphine

TMPO trimethylphosphine oxide

TOPO trioctylphosphine oxide

TPA tetrapropylammonium

TPD temperature-programmed desorption

TPP triphenylphosphine

TQ Triple quantum

TRAPDOR transfer of population in double resonance

USY ultra-stable Y type zeolite

UV-vis ultraviolet-visible

vdW van der Walls

XPS X-ray photoelectron spectroscopy

XRD X-ray diffraction

XRF X-ray fluorescence

ZSM-5 Zeolite Socony Mobil-5

\section{Solid-state NMR spectroscopy}

\subsection{A brief introduction ${ }^{1,2}$}

The application of nuclear magnetic resonance (NMR) spectroscopy to solution-state samples exists since the 1960's. However, the rapid development of hardware and instrumentations as well as the methodological and technical advances led to the successful application of NMR to samples in solid state. Last four decades saw an explosion in the use of solid-state NMR (ssNMR) to a very wide range of samples. These days, scientists routinely make use of the ssNMR interactions (chemical shift interactions, dipole-dipole interactions, quadrupole interactions, and indirect spin-spin (j) coupling) to determine the molecular structure, conformation and dynamics. It is a fast, non-destructive, accurate, quantitative and high resolution tool for the characterization of solids at the molecular level. 
Nuclear magnetic resonance is concerned with the magnetic moment of atomic nuclei with a nuclear spin $I \neq 0$. If those atomic nuclei are placed in an external magnetic field, the nuclear spin interacts with field and is called Zeeman interaction. The magnetic field at the nuclei is not equal to the applied external magnetic field, surrounding nuclei and electrons shield it from the applied field. Nuclear magnetic resonance spectroscopy is based on the interactions of the nuclear magnetic moment with an electromagnetic field (radio frequency range) while a strong external magnetic field is applied. While the Zeeman interaction is useful in identifying the nuclei placed in a magnetic field, other magnetic and electronic interactions are responsible for acquiring the information on structure and dynamics.

\subsection{Chemical shift interactions}

The most commonly explored interaction in ssNMR is the chemical shift. The shielding or deshielding of the nucleus by its surrounding valence electrons is named chemical shift. Information on coordination environment, bonding to different atoms, bond related parameters, bond types etc. are provided by isotropic chemical shift $\left(\delta_{\text {iso }}\right)$. In the solid-state, restricted mobility leads to asymmetry in the chemical shift (chemical shift anisotropy - CSA) which results in broad line shapes. The analysis of such line shapes gives additional information on the geometry and motion. Various experimental investigations, designs with multiple independent NMR active nuclei, are outlined in this report.

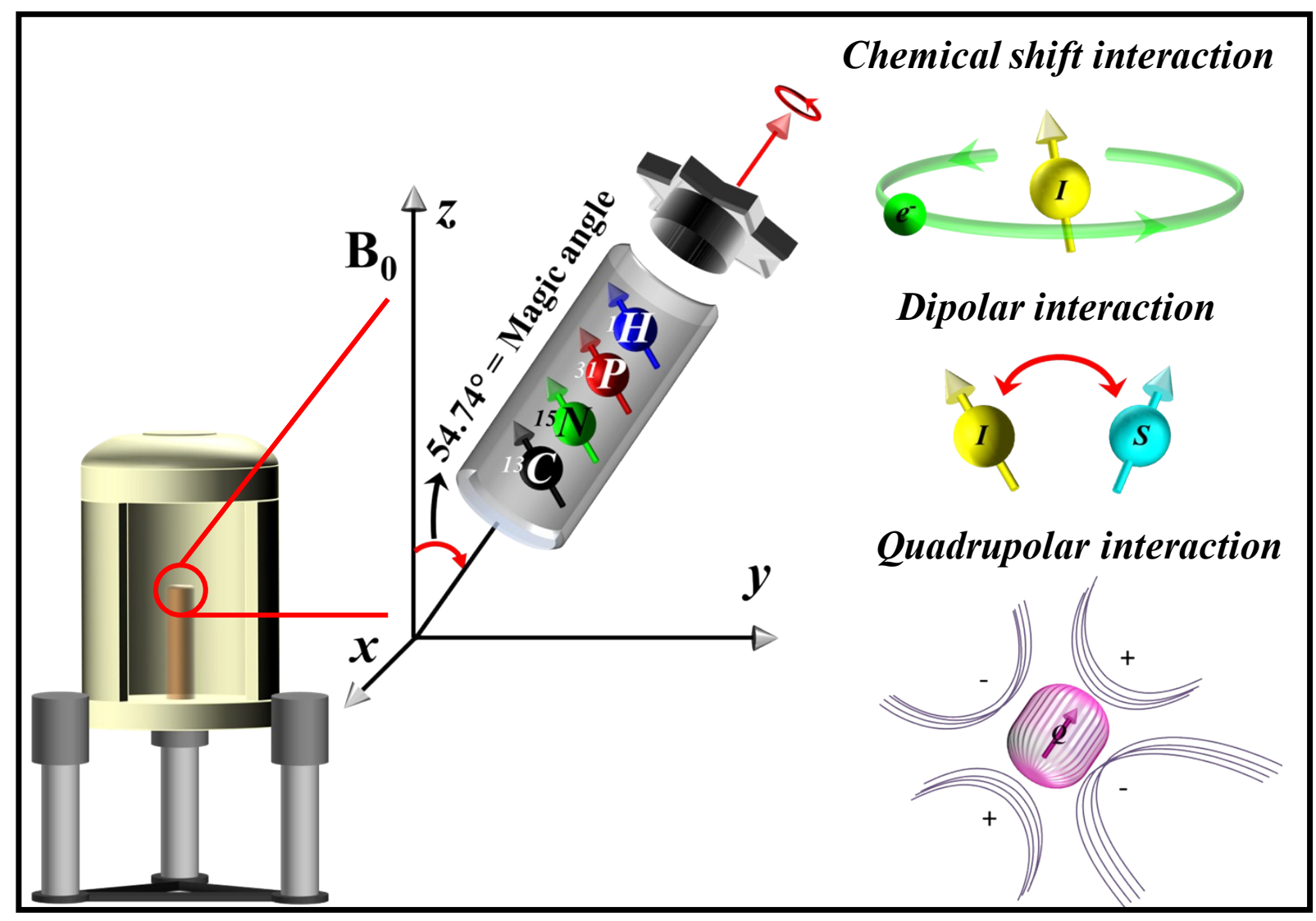

Fig. 1 Schematics of a solid-state NMR super conducting magnet and MAS probe with a depiction of magic angle rotor spinning, chemical shift interaction, dipolar interaction and quadrupolar interaction. Figure in part 
inspired and adapted from $\quad$ M. Levitt's $\quad$ webpage

(http://www.southampton.ac.uk/ mhl/publications/books/SpinDynamics/SD1/index.html)

\subsection{Dipolar interactions}

Through-space magnetic interaction of two NMR active nuclei, either of same kind (homonuclear) or of different kind (heteronuclear), is called dipolar interactions, which depends on the inter-nuclear distance. In the solid-state, dipolar interactions are easily observed when the other interactions are negligible and is a spectroscopic path to determine inter-nuclear distances. There are four major factors that determine the strength of the dipolar interactions: types of nuclei, distance and angle between them as well as their relative motion. Several ssNMR experiments exploiting this interaction are sketched throughout this review.

\subsection{Quadrupolar interactions}

A majority of ssNMR active nuclei are quadrupolar $(\mathrm{I}>1 / 2)$ in nature. Besides the nuclear magnetic moment, they also possess nuclear electric quadrupole moment. This quadrupolar moment interacts with electric field gradients (EFG) generated by uneven charge distribution within the nucleus and is called quadrupolar interactions. The strength of the interaction depends upon the magnitude of the nuclear quadrupolar moment (property of the nuclei) and the strength of the EFG (depends on molecular environment). The magnitude of the broadening can mask any information about chemical shift or dipolar couplings. For nuclei in a highly symmetric environment (e.g.: cubic), EFG tends to be zero, therefore, behaves like a spin-half nuclei. Information on local symmetry is provided by quadrupolar coupling constant $\left(C_{q}\right)$ and asymmetry parameter $(\eta)$. This review will demonstrate how experiments on quadrupolar nuclei can be helpful in revealing molecular-level information from porous solids.

\subsection{Indirect nuclear spin-spin (j) couplings}

The magnetic interaction between nuclear spins (homonuclear or heteronuclear) mediated by the electrons of the covalent bonds is called indirect nuclear spin-spin (j) coupling or scalar coupling. The indirect coupling originates from the perturbation of the electronic system due to the magnetic hyperfine interaction with one nucleus that leads to a change in the hyperfine interaction of the electrons with the other nuclei. Although they are present in solid state, its influence is small compared to dipolar interactions and are rarely resolved. However, with the development of advanced techniques, it is possible to discriminate between through-bond (scalar coupling) and through space (dipolar coupling) connectivities in solids. Indeed, indirect spin-spin couplings based experiments are proven to be important in solids were dipolar-driven correlation fails due to motions. Such studies are useful in assigning the structural and connectivity related information of porous solids. 
Powdered sample comprises of various crystallites with random orientations with respect to the external magnetic field. Most important NMR interactions such as chemical shielding, dipolar and quadrupolar interactions are anisotropic in nature (i.e., orientation dependent). As a result, static NMR spectrum of powdered sample is a summation of the individual contributions from each crystallite which results in broad lines. Anisotropic interactions can be averaged to zero if the powdered sample is physically rotated at an angle of $54.736^{\circ}$ (magic angle) relative to the orientation of the external magnetic field. ${ }^{3}$ When the powdered sample is subjected to magic angle spinning (MAS) at a rate faster than the anisotropy of the interactions, all the crystallites appear to have the same orientation (isotropic) and results in a high resolution solid-state NMR spectrum. ${ }^{4}$ Rapid isotropic molecular tumbling in solutionstate averages out the anisotropic interactions, resulting in narrow spectral lines in liquid samples. Magic angle spinning rates up to $110 \mathrm{kHz}$ are possible in commercially available probes today.

\subsection{Cross Polarization (CP)}

The disadvantage of directly detecting low $\gamma$ nuclei such as ${ }^{15} \mathrm{~N}$ or ${ }^{13} \mathrm{C}$ are low isotopic abundances, low spin polarization, and low signal intensity. These weaknesses can be circumvented by a solid-state NMR technique that combines both the high polarization and short relaxation time. The enhancement of the signals from rare nuclei $\left({ }^{15} \mathrm{~N}\right.$ or ${ }^{13} \mathrm{C}$ etc) involve the transfer of polarization from abundant nuclei $\left({ }^{1} \mathrm{H}\right.$ or ${ }^{19} \mathrm{~F}$ etc) by using cross polarization $(\mathrm{CP}){ }^{5}$ The process of $\mathrm{CP}$ occurs through the tendency of the magnetization to flow from highly polarized nuclei (for e.g., ${ }^{1} \mathrm{H}$ ) to less polarized nuclei (for e.g., ${ }^{13} \mathrm{C}$ ) when they are brought into contact and depends on the CP contact time and dipolar coupling strength. The exchange of magnetization must be driven externally by the application of radio frequency fields.

\section{Probe molecules}

Multinuclear NMR spectroscopy $\left({ }^{1} \mathrm{H},{ }^{13} \mathrm{C},{ }^{15} \mathrm{~N}\right.$, and $\left.{ }^{31} \mathrm{P}\right)$ can be applied for the investigation of the probe molecules perturbed by surface hydroxyl sites. However, rapid thermal motions and chemical exchanges at room temperature in probe adsorbed porous systems have to be taken into account in these studies. Alternatively, Lewis acidic sites can be directly detected by MAS $\left({ }^{27} \mathrm{Al},{ }^{119} \mathrm{Sn}\right.$ and $\left.{ }^{47,49} \mathrm{Ti}\right)$ NMR spectroscopy, which can distinguish between framework and extra-framework species. ${ }^{6-8}$ Integrated ${ }^{1} \mathrm{H}$ MAS and FT-IR spectroscopic study of porous solids using probe molecules gives complimentary information on the concentration and strength of acidity as well as the accessibility of acid sites. Although a variety of probe molecules, either polynuclear or binuclear, are available today, strong bases are not employed for the study of heterogeneity of Brønsted acids. ${ }^{9}$

Solid-state NMR spectroscopy has the ability and potential for the characterization of Brønsted acid and Lewis acid sites, simultaneously, on a solid catalyst as have been demonstrated in the past decades. ${ }^{10,11}$ Often, the ${ }^{13} \mathrm{C}$ NMR shift of the carbonyl atom of 
adsorbed acetone-2-13C was utilized for characterizing the nature and strength of Brønsted and Lewis acid sites on porous solids. ${ }^{12,13}$ In a recent report, a correlation between the ${ }^{13} \mathrm{C}$ chemical shift of adsorbed acetone-2- ${ }^{13} \mathrm{C}$ and intrinsic Brønsted acidic strength has been deduced. ${ }^{14}{ }^{13} \mathrm{C}$ chemical shift range can be used as a scale for quantitative measurement of acidic strength in both Lewis acid and Brønsted acid sites (Fig. 2). ${ }^{13} \mathrm{C}$ chemical shift of acetone-2- ${ }^{13} \mathrm{C}$ adsorbed on silanols would appear at around $210 \mathrm{ppm}$ while on the Brønsted acid site in the range $215-235 \mathrm{ppm}$ and on Lewis acid sites in the range $235-245$ ppm. ${ }^{15}$

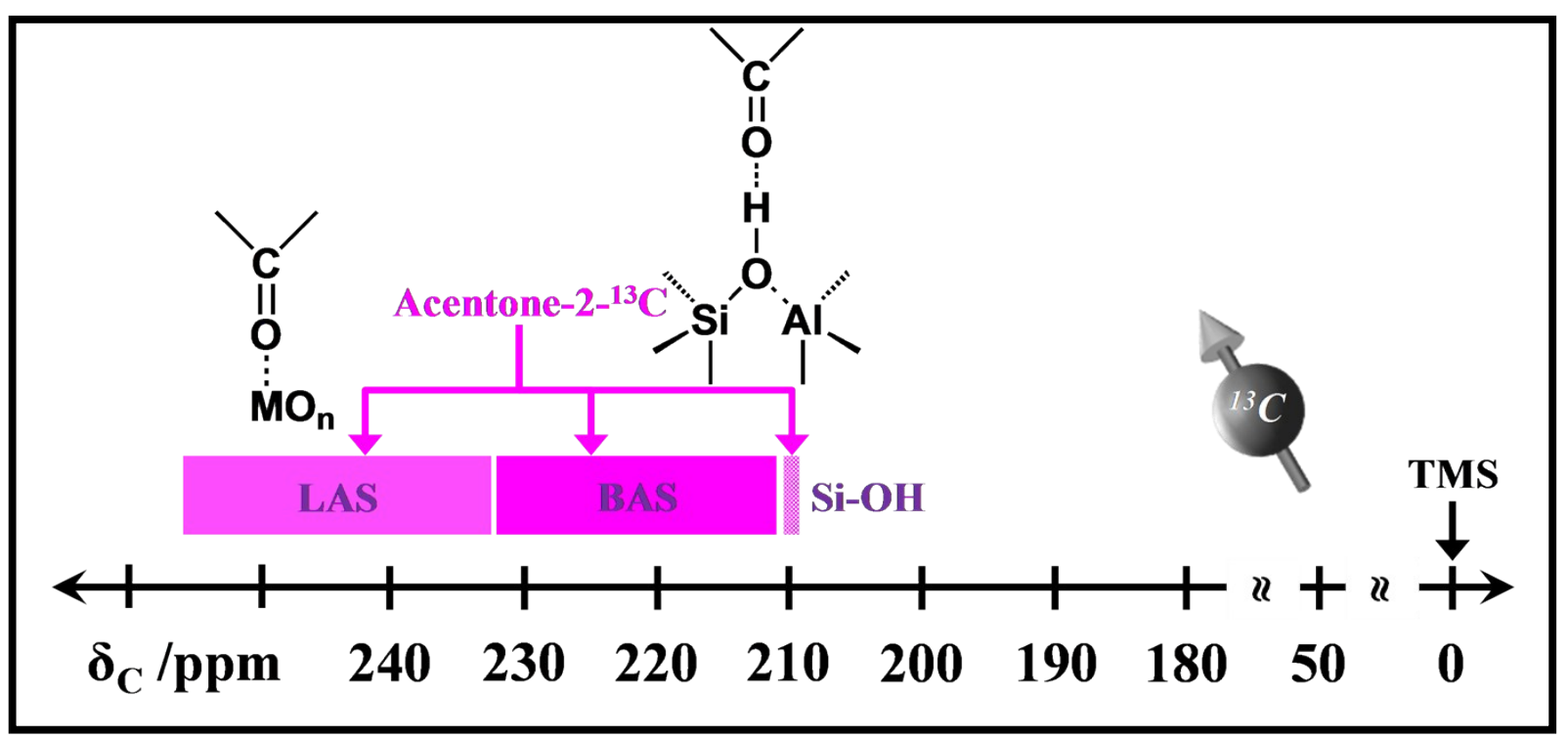

Fig. $2{ }^{13} \mathrm{C}$ MAS NMR chemical shift scale of ${ }^{13} \mathrm{C}$ environments for carbonyl carbon related to acetone adsorption. LAS; Lewis acid site, BAS; Brønsted acid site, $M O_{n}$; metal oxide site.

It has been demonstrated recently that ${ }^{31} \mathrm{P}$ MAS NMR chemical shifts of various phosphorous based molecular probes can be used as a tool for the variable acid strength characterization of solid catalysts. ${ }^{16}{ }^{31} \mathrm{P}$ based probe molecules include various trialkylphosphine oxides (trimethylphosphine oxide; TMPO, triethylphosphine oxide; TEPO, tributylphosphine oxide; TBPO and trioctylphosphine oxide; TOPO) and trialkylphosphines (trimethylphosphine; TMP and triphenylphosphine, TPP). Chemical shift of TMPO is highly sensitive to the acid strength and can be introduced to porous systems in gas phase or liquid phase using solvents. In a recent report, ${ }^{17}$ Hayashi et al., studied the acid properties of ZSM-5 type zeolite by solidstate NMR using TMPO as a probe molecule. They have introduced TMPO from the gas phase at $373 \mathrm{~K}$ in order to increases the loading level as well as to probe almost all the acid cites. ${ }^{31} \mathrm{P}$ MAS NMR chemical shifts scale of selected probe molecules are shown in Fig. 3. 


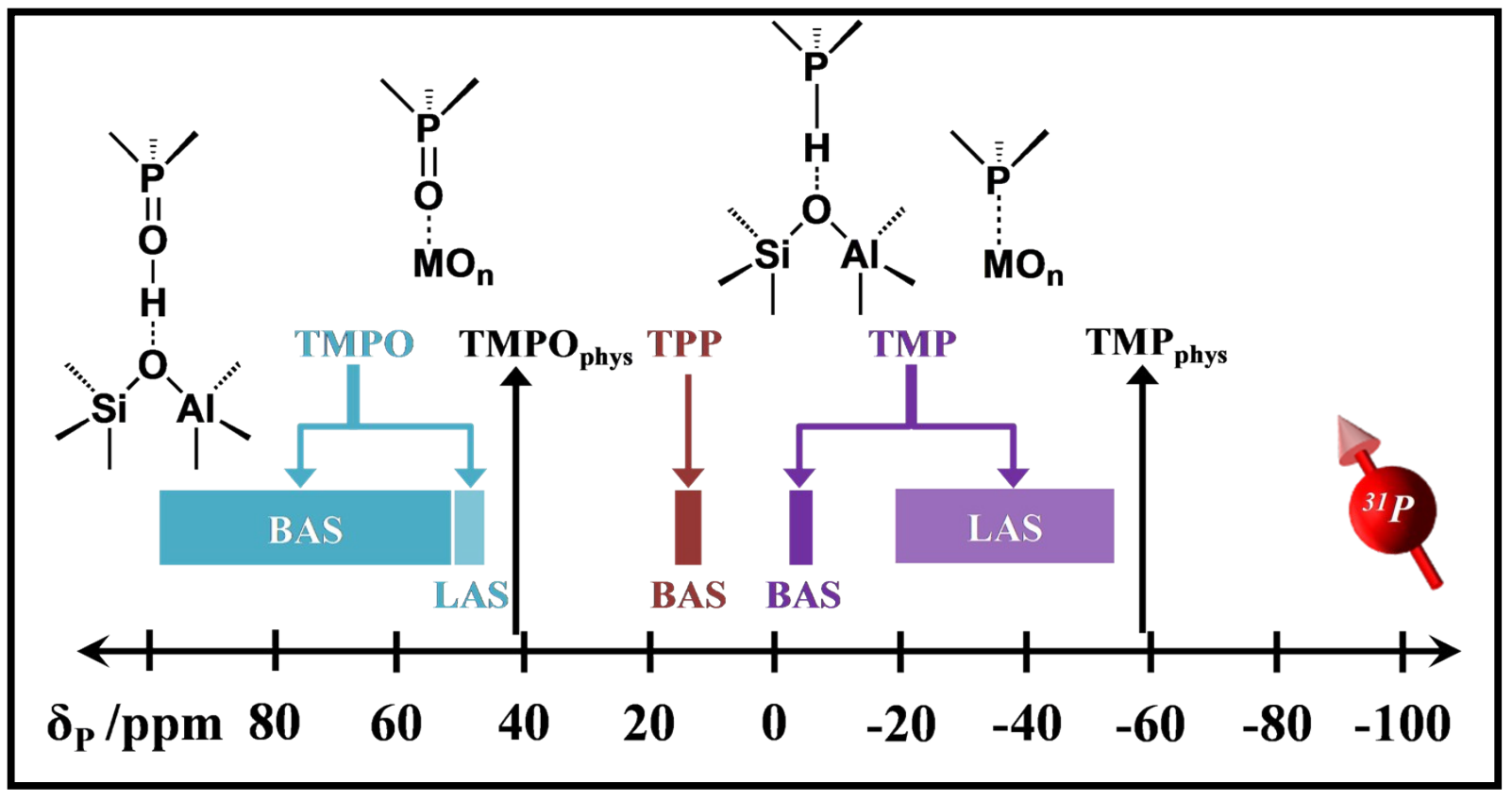

Fig. $3{ }^{31} P$ MAS NMR chemical shift scale of selected ${ }^{31} P$ environments related to probe adsorption. BAS; Brønsted acid site, LAS; Lewis acid site, TMPO; Trimethylphosphine oxide, TPP; triphenylphosphine, TMP; trimethylphosphine, TMPO phys; physisorbed TMPO, TMP phys; physisorbed TMP, $M O_{n}$; metal oxide site.

\section{Glossary}

\subsection{DNP NMR}

Dynamic Nuclear Polarisation experiment ${ }^{18}$ is based on the transfer of spin polarization from electrons to NMR active nuclei to enhance the nuclear spin polarisation and eventually better sensitivity. Larger polarization of electron spins can be transferred to nuclear spins upon saturation of electron paramagnetic resonance transitions by means of microwave irradiation. State of the art of solid-state DNP NMR instrumentation and its applications has been recently reviewed. ${ }^{19,20}$

\section{2. $D Q-S Q$ and $T Q-S Q$}

Multiple quantum transitions are quantum-mechanically forbidden for direct observation, but they can be made visible in a $2 \mathrm{D}$ experiment showing single quantum (SQ) chemical shifts in one and double quantum (DQ) or triple Quantum (TQ) chemical shifts in the other dimension. These transitions can be promoted in RF pulse sequences to identify or select pairs of nuclei (for DQ) or cluster of three nuclei (for TQ) that are covalently bonded (indirect nuclear spin-spin $(\mathrm{J})$ couplings) or spatially close (dipolar couplings). 


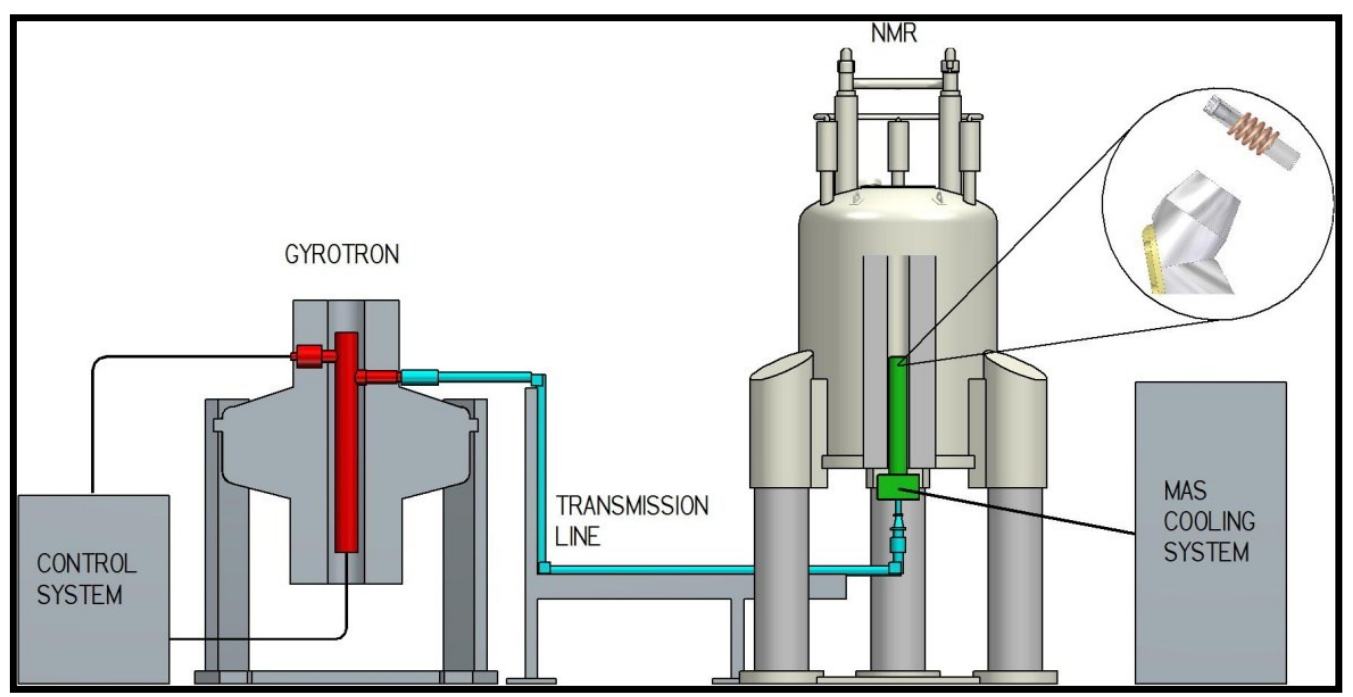

Fig. 4 Schematics of a solid-state DNP NMR system with a gyrotron microwave source (gyrotron tube in red), microwave transmission line (cyan) and low-temperature NMR probe (green). Reprinted with permission from M. Rosay, M. Blank and F. Engelke, Journal of Magnetic Resonance, 2016, 264, 88-98. Copyright 2016 Elsevier Ltd.

\subsection{HETCOR}

HETeronuclear CORrelation technique probes the heteronuclear dipolar interactions, whose strength decreases with increasing inter-nuclear distance. Signals in the two-dimensional spectrum appear only when both nuclei are in close proximity. HETCOR technique can be applied in a combined strategy to generate a link between three connectivity partners ${ }^{21}$ (e.g. ${ }^{1} \mathrm{H}-{ }^{29} \mathrm{Si}-{ }^{13} \mathrm{C} ;{ }^{1} \mathrm{H}-{ }^{29} \mathrm{Si}$ correlation and ${ }^{1} \mathrm{H}-{ }^{13} \mathrm{C}$ correlation).

\section{4. $M Q M A S$}

Multiple-Quantum Magic Angle Spinning experiment ${ }^{22}$ allows the separation of isotropic and anisotropic quadrupolar information in two-dimensional spectra of half-integer quadrupolar nuclei. It correlates multiple- and single-quantum coherences in the presence of MAS. 
The Rotational Echo DOuble Resonance ${ }^{23}$ is a high-resolution experiment based on the reintroduction of heteronuclear dipolar interaction under MAS. By measuring the dipolar coupling between a heteronuclear spin pair, one can estimate the distance between the spin pair.

\subsection{S-RESPDOR}

The Symmetry-based Resonance-Echo Saturation-Pulse DOuble-Resonance (S-RESPDOR) experiment ${ }^{24}$ is used to measure the inter-nuclear distances between spin-1/2 and quadrupolar nuclei. This sequence employs a symmetry-based recoupling scheme on the observed spin$1 / 2$ channel and a saturation pulse on the quadrupolar channel.

\subsection{Spin-echo}

In a basic spin-echo experiment ${ }^{25}$ on nuclear spins, the single- and multiple-quantum coherences generated by the first pulse are refocused as echoes by the second pulse with a delay $\tau$ in between.

\subsection{TRAPDOR}

TRAnsfer of Population in DOuble Resonance experiment ${ }^{26}$ is based on heteronuclear dipole coupling between quadrupolar and spin-1/2 nuclei and is used to establish connectivity and spatial interaction information between nuclei. It is based on a spin-echo performed on spin$1 / 2$ nuclei combined with continuous irradiation applied alternatively on the quadrupolar spin.

\section{9. $T_{1}, T_{2}$}

Spin-lattice relaxation time or longitudinal relaxation time $\left(\mathrm{T}_{1}\right)$, Spin-spin relaxation time or transverse relaxation time $\left(\mathrm{T}_{2}\right)$. 


\section{References}

1 M. H. Levitt, Spin dynamics: basics of nuclear magnetic resonance, John Wiley \& Sons, Chichester, England; Hoboken, NJ, 2nd ed., 2008.

2 M. J. Duer, Introduction to solid-state NMR spectroscopy, Blackwell, Oxford, UK; Malden, MA, 2004.

3 E. R. Andrew, A. Bradbury and R. G. Eades, Nature, 1959, 183, 1802-1803.

4 E. R. Andrew and E. Szczesniak, Prog. Nucl. Magn. Reson. Spectrosc., 1995, 28, $11-$ 36.

$5 \quad$ S. R. Hartmann and E. L. Hahn, Phys. Rev., 1962, 128, 2042-2053.

6 S. Ganapathy, K. U. Gore, R. Kumar and J.-P. Amoureux, Solid State Nucl. Magn. Reson., 2003, 24, 184-195.

7 R. Bermejo-Deval, M. Orazov, R. Gounder, S.-J. Hwang and M. E. Davis, ACS Catal., 2014, 4, 2288-2297.

8 J. Brus, L. Kobera, W. Schoefberger, M. Urbanová, P. Klein, P. Sazama, E. Tabor, S. Sklenak, A. V. Fishchuk and J. Dědeček, Angew. Chem. Int. Ed., 2015, 54, 541-545.

9 C. Paze', A. Zecchina, S. Spera, G. Spano and F. Rivetti, Phys. Chem. Chem. Phys., 2000, 2, 5756-5760.

10 M. Hunger, Catal. Rev., 1997, 39, 345-393.

11 G. Ertl, Ed., Handbook of heterogeneous catalysis: 8 volumes, WILEY-VCH, Weinheim, 2., completely rev. and enl. ed., 2008.

12 Y. Jiang, J. Huang, W. Dai and M. Hunger, Solid State Nucl. Magn. Reson., 2011, 39, $116-141$.

13 A. Zheng, S. Li, S.-B. Liu and F. Deng, Acc. Chem. Res., 2016, 49, 655-663.

14 H. Fang, A. Zheng, Y. Chu and F. Deng, J. Phys. Chem. C, 2010, 114, 12711-12718.

15 S. Lang, M. Benz, U. Obenaus, R. Himmelmann, M. Scheibe, E. Klemm, J. Weitkamp and M. Hunger, Top. Catal., 2017, 60, 1537-1553.

16 A. Zheng, S.-B. Liu and F. Deng, Chem. Rev., 2017, 117, 12475-12531.

17 S. Hayashi, K. Jimura and N. Kojima, Microporous Mesoporous Mater., 2014, 186, 101-105.

18 L. R. Becerra, G. J. Gerfen, R. J. Temkin, D. J. Singel and R. G. Griffin, Phys. Rev. Lett., 1993, 71, 3561-3564.

19 M. Rosay, M. Blank and F. Engelke, J. Magn. Reson., 2016, 264, 88-98.

20 A. J. Rossini, A. Zagdoun, M. Lelli, A. Lesage, C. Copéret and L. Emsley, Acc. Chem. Res., 2013, 46, 1942-1951.

21 G. Paul, S. Steuernagel and H. Koller, Chem. Commun., 2007, 5194-5196.

22 A. Medek, J. S. Harwood and L. Frydman, J. Am. Chem. Soc., 1995, 117, 1277912787.

23 T. Gullion and J. Schaefer, J. Magn. Reson., 1989, 81, 196-200.

24 L. Chen, X. Lu, Q. Wang, O. Lafon, J. Trébosc, F. Deng and J.-P. Amoureux, J. Magn. Reson., 2010, 206, 269-273.

25 H. Y. Carr and E. M. Purcell, Phys. Rev., 1954, 94, 630-638.

26 C. P. Grey and W. S. Veeman, Chem. Phys. Lett., 1992, 192, 379-385. 